\title{
Cerulenin-induced apoptosis is mediated by disrupting the interaction between AIF and hexokinase II
}

\author{
NA YOUNG JEONG and YOUNG HYUN YOO
}

\author{
Department of Anatomy and Cell Biology and Mitochondria Hub Regulation Center, \\ Dong-A University College of Medicine, Busan, Republic of Korea
}

Received December 22, 2011; Accepted February 28, 2012

DOI: $10.3892 /$ ijo.2012.1401

\begin{abstract}
Fatty acid synthase (FASN) is a key enzyme that plays a critical role in numerous metabolic functions by catalyzing the synthesis for long-chain fatty acids. FASN is highly expressed in various human cancers. This preferential expression makes FASN an attractive target for anticancer therapy. Hexokinase II (HKII) is overexpressed in most cancer cells, and it generally localizes to the outer mitochondrial membrane. Recent studies have demonstrated the protective role of mitochondrial HKII in preservation of mitochondrial integrity. The association of hexokinase with mitochondria has emerged as a powerful mechanism in protecting numerous cell types against cell death. We performed this study to examine the mechanism underlying apoptosis induced by cerulenin and with specific focus on its effect on HKII in ZR-75-1 human breast cancer cells. Additionally, we sought to elucidate whether inhibition of the PI3K/Akt pathway can potentiate the anticancer effect of cerulenin. Here, we showed that cerulenin disrupts the physical association between HKII and AIF, leading to eventual cell death. In addition, LY294002, a PI3K/Akt inhibitor, sensitized ZR-75-1 breast cancer cells to cerulenin-induced apoptosis. Collectively, cerulenin induces apoptosis via disrupting the interaction between AIF and HKII and inhibition of PI3K sensitizes cells to cerulenin-induced apoptosis in ZR-75-1 cells.
\end{abstract}

\section{Introduction}

Fatty acid synthase (FASN) is a key enzyme that plays a critical role in numerous metabolic functions by catalyzing the synthesis for long-chain fatty acids (1). In normal cells, FASN expression level is found to be low because of the presence of abundant amounts of dietary fat (1). However, there is rejuvenated interest in the ultimate role of FASN in cancer pathogenesis. FASN is

Correspondence to: Dr Young Hyun Yoo, Department of Anatomy and Cell Biology, Dong-A University College of Medicine and Mitochondria Hub Regulation Center, 3-1 Dongdaesin-dong, Seo-gu, Busan 602-714, Republic of Korea

E-mail: yhyoo@dau.ac.kr

Key words: fatty acid synthase, cerulenin, hexokinase II, AIF, Akt highly expressed in many types of human cancers, including breast, colon, ovary, lung and prostate $(1,2)$. Increased expression of FASN occurs very early in cancer development and is more pronounced in clinically aggressive cancers (3). This preferential expression makes FASN an attractive target for anticancer therapy. Inhibition of FASN activity preferentially inhibits tumor cell growth and induces apoptosis in breast cancer (5), prostate cancer (6), and mesothelioma cells (7). Cerulenin [(2S,3R)]-23-epoxy-4-oxo-7,10-dodecadienoylamide), a fungal metabolite, is a specific inhibitor of fatty acid synthase (8). Cerulenin treatment showed regression of established ascites tumor, reduction in ascites incidence, delay in onset of ascites, and significantly increased survival in a nude mouse xenograft model of human ovarian carcinoma (9).

Substantial evidence shows that phosphatidylinositol 3-kinase(PI3K)/Akt signaling plays an essential role in oncogenic transformation and cancer progression and has been linked with FASN expression in tumor cells $(10,11)$. PI3K/Akt signaling has been implicated in regulating FASN expression in breast cancer cells (12), prostate cancer cells (13), and adipocyte/3T3 cells (14). In addition, inhibition of FASN activity causes dephosphorylation of Akt and apoptosis (11). Akt confers mitochondrial protection through phosphorylation of the proapoptotic Bcl-2 family protein Bad (15). Interestingly, the ability of Akt to protect against cytochrome c release and apoptosis in fibroblasts can be inhibited by hexokinase II (HK II) dissociation from mitochondria (16).

Hexokinases play an important role in the cellular uptake and utilization of glucose (17). Hexokinases catalyse the essentially irreversible first step of the glycolytic pathway where glucose is phosphorylated to glucose-6-phosphate using ATP as phosphodonor (18). Four highly homologous hexokinase isoforms, denoted here as HKI, II, III and IV (glucokinase), have been described in mammalian tissues (19). Individual isoforms share a number of close structural and functional similarities that have been ascribed to a common evolutionary origin (20). They are also classified by critical differences in kinetic features, regulatory properties, intracellular distribution and patterns of expression that purposedly fit individual isoforms for distinct metabolic roles within cells (19). Hexokinases I and II (HKI and HKII) have mitochondrial binding motifs and these enzymes have been found at mitochondria as well as cytosol (21). HKII has an Akt phosphorylation consensus sequence (RxRxxS/T) (Thr473) and serves as a substrate for phosphorylation by 
Akt (22). Activated Akt increases association of hexokinase with mitochondria (23). The first committed step in glucose metabolism catalyzed by hexokinase is sufficient for Akt to inhibit apoptosis (23). Mitochondrial-bound hexokinase is a downstream effector of Akt-mediated cell survival (23).

We performed this study to examine the mechanism underlying apoptosis induced by cerulenin in ZR-75-1 human breast cancer cells. Here, we show that cerulenin induces apoptosis via disrupting interaction between AIF and HKII and that inhibition of PI3K sensitizes cerulenin-induced apoptosis in ZR-75-1 cells.

\section{Materials and methods}

Reagents. Rabbit polyclonal anti-human Akt, phospho-Akt (s473), Bcl-2, Bcl-xL, cIAP-1, Mcl-1, cytochrome c, and AIF antibodies were obtained from Santa Cruz Biotechnology (Santa Cruz, CA). Rabbit polyclonal anti-human hexokinase II, fatty acid synthase and caspase- 3 antibodies were obtained from Cell Signaling Technology (Danvers, MA). Fluorescein isothiocyanate (FITC)-conjugated goat anti-rabbit and horse anti-mouse IgG antibodies were obtained from Vector (Burlingame, CA). HRP-conjugated donkey anti-rabbit and sheep anti-mouse IgG antibodies were obtained from Amersham Pharmacia Biotech (Piscataway, NJ).3,3'-dihexyloxa carbocyanine iodide (DIOC6) and Mitotracker was obtained from Molecular Probes (Eugene, OR). RPMI-1640 and fetal bovine serum (FBS) were obtained from Gibco (Gaithersburg, MD). The enhanced chemiluminescent western blotting detection reagent (SuperSignal West Pico chemiluminescent substrate) was obtained from Pierce (Rockford, IL). Cerulenin and propidium iodide were obtained from Sigma (St. Louis, MO). LY294002 was obtained from Calbiochem (San Diego, CA).

Cell culture.ZR-75-1 human breast cancer cells were purchased from the American Type Culture Collection (ATCC, Rockville, MD). The culture medium used throughout these experiments was RPMI-1640 medium, containing $10 \%$ fetal calf serum (FCS), $20 \mathrm{mM}$ HEPES buffer, $100 \mathrm{U} / \mathrm{ml}$ penicillin and $100 \mathrm{U} /$ $\mathrm{ml}$ streptomycin.

Cerulenin and LY294002 treatment and assessment of cell viability. Stock solutions were prepared in dimethyl sulfoxide (DMSO) and stored at $-20^{\circ} \mathrm{C}$. Before each experiment, drugs were diluted in fresh media. The final DMSO concentration was $<0.1 \%$ for all experiments. Cells were treated with cerulenin at $0-10 \mu \mathrm{g} / \mathrm{ml}$ or LY294002 at 0-6.1 $\mu \mathrm{g} / \mathrm{ml}$. Cells were harvested $48 \mathrm{~h}$ after treatment and cell viability was determined with the Vi-Cell cell counter (Beckman Counter, Fullerton, CA), which performs an automated trypan blue exclusion assay.

Flow cytometric analysis. Ice-cold $95 \%$ ethanol with $0.5 \%$ Tween-20 was added to cell suspension to a final concentration of $70 \%$ ethanol. Fixed cells were pelleted and washed in $1 \%$ BSA-PBS solution. Cells were re-suspended in $1 \mathrm{ml}$ PBS containing $11 \mathrm{Kunitz} \mathrm{U} / \mathrm{ml} \mathrm{RNase,} \mathrm{incubated} \mathrm{at} 4^{\circ} \mathrm{C}$ for $30 \mathrm{~min}$, washed once with BSA-PBS, and re-suspended in PI solution $(50 \mu \mathrm{g} / \mathrm{ml})$. After cells had been incubated at $4^{\circ} \mathrm{C}$ for $30 \mathrm{~min}$ in the dark and washed with PBS, DNA content was measured on an Epics XL (Beckman Coulter, FL, USA), and data were analyzed using Multicycle software, which allowed a simultaneous estimation of cell cycle parameters and apoptosis.

Nuclear morphology analysis of apoptosis. Forty-eight hours after treatment, the cell suspension was cytospun onto a clean fatfree glass slide with a cytocentrifuge. Cytocentrifuged samples were fixed for $10 \mathrm{~min}$ in $4 \%$ paraformaldehyde and stained in $4 \mu \mathrm{g} / \mathrm{ml}$ Hoechst 33342 for $30 \mathrm{~min}$ at $4^{\circ} \mathrm{C}$. The total cell number (300 cells from each experiment) was counted using differential interference contrast (DIC) optics, and the number of cells showing condensed or fragmented nuclei on Hoechst staining was calculated using epifluorescence optics, by an observer who was blinded with regard to the experimental group.

Western blot analysis. Cells $\left(2 \times 10^{6}\right)$ were washed twice in icecold PBS, resuspended in $200 \mu \mathrm{l}$ ice-cold solubilizing buffer [300 mM NaCl, $50 \mathrm{mM}$ Tris-Cl (pH 7.6), 0.5\% Triton X-100, $2 \mathrm{mM}$ phenylmethylsulfonyl fluoride (PMSF), $2 \mu \mathrm{l} / \mathrm{ml}$ a protin and $2 \mu \mathrm{l} / \mathrm{ml}$ leupeptin] and incubated at $4^{\circ} \mathrm{C}$ for $30 \mathrm{~min}$. The lysates were centrifuged at $14,000 \mathrm{rpm}$ for $15 \mathrm{~min}$ at $4^{\circ} \mathrm{C}$. Protein concentrations of cell lysates were determined with Bradford protein assay (Bio-Rad, Richmond, CA) and $30 \mu \mathrm{g}$ of proteins was loaded onto 7.5-15\% SDS-polyacrylamide gels. The gels were transferred to nitrocellulose membrane (Amersham Pharmacia Biotech, Piscataway, NJ) and reacted with each antibody. Immunostaining with antibodies was performed using SuperSignal West Pico enhanced chemiluminescence substrate and detected with LAS-3000 PLUS (Fuji Photo Film Co., Kanagawa, Japan).

Assay of mitochondrial membrane potential (MMP). 3,3'-dihexyloxa carbocyanine iodide (DIOC6) was added directly to the cell culture medium ( $1 \mu \mathrm{M}$ final concentration) and incubated for $15 \mathrm{~min}$. The medium was then replaced with PBS, and cells were resuspended in $10 \mu \mathrm{g} / \mathrm{ml}$ of methanol and incubated at $37^{\circ} \mathrm{C}$ for $30 \mathrm{~min}$. Flow cytometry to measure MMP was performed on a Epics XL (Beckman Coulter, FL, USA). Data were acquired and analyzed using EXPO32 ADCXL 4 color software. The analyzer threshold was adjusted on the forwards catter (FSC) channel to exclude noise and most of the subcellular debris.

Immunofluorescent staining and confocal microscopy. A cell suspension was cytospun onto a clean fat-free glass slide with a cytocentrifuge. Cells were incubated with each primary antibody for $1 \mathrm{~h}$, washed 3 times for $5 \mathrm{~min}$ each and then incubated with FITC-conjugated or Texas Red-conjugated secondary antibodies for $1 \mathrm{~h}$ at room temperature. Fluorescent images were observed and analyzed under Zeiss LSM 510 laser-scanning confocal microscope (Zeiss, Goettingen, Germany).

Isolation of mitochondria and protein fractionation. Cells $\left(5 \times 10^{7}\right)$ were washed in Tris-based, $\mathrm{Mg}^{2} / \mathrm{Ca}^{2}$-deficient buffer [135 mM NaCl, $5 \mathrm{mM} \mathrm{KCl}$, and $25 \mathrm{mM}$ Tris-Cl (pH 7.6)] and allowed to swell for $10 \mathrm{~min}$ in ice-cold hypotonic CaRSB buffer [10 mM NaCl, $1.5 \mathrm{mM} \mathrm{CaCl}_{2}, 10 \mathrm{mM}$ Tris- $\mathrm{HCl}$ (pH 7.5), and 1 protease inhibitor cocktail]. Cells were dounced with 60 strokes, and mitochondria stabilization buffer [210 $\mathrm{mM}$ mannitol, 70 $\mathrm{mM}$ sucrose, $5 \mathrm{mM}$ EDTA, and $5 \mathrm{mM}$ Tris (pH 7.6)] was added to stabilize mitochondria ( $2 \mathrm{ml}$ of 2.5 per $3 \mathrm{ml}$ of homogenate). After collecting the nucleus (by centrifuging twice at 3,000 
A

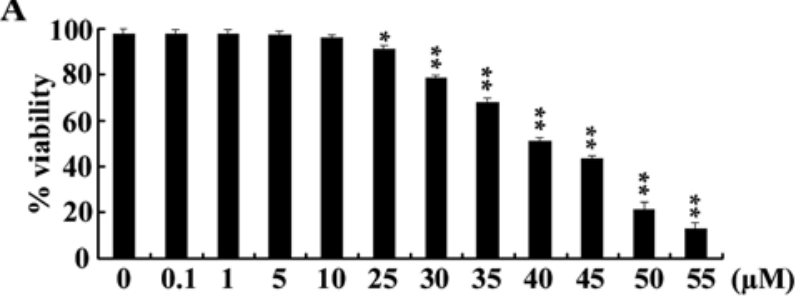

B

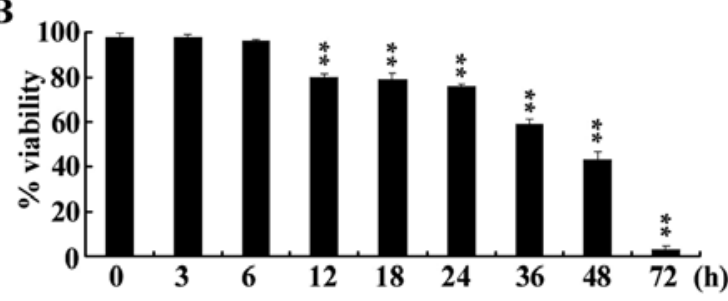

Figure 1. Cerulenin reduces viability of ZR-75-1 cells in a dose- and timedependent manner. (A) Cells were harvested $48 \mathrm{~h}$ after treatment with cerulenin. (B) ZR-75-1 cells were treated with $45 \mu \mathrm{M}$ cerulenin and viability was determined automated trypan blue exclusion assay with a cell counter. ${ }^{*} \mathrm{P}<0.05$ and ${ }^{* *} \mathrm{P}<0.01$ versus untreated control.

revolutions per minute for $15 \mathrm{~min}$ ), the supernatant was spun at $14,000 \mathrm{rpm}$ for $20 \mathrm{~min}$ at $4^{\circ} \mathrm{C}$. The pellet and the supernatant included mitochondria and cytoplasm, respectively.

Co-immunoprecipitation (Co-IP). Cell extracts that were incubated with antibodies were precipitated with protein A-Sepharose beads. Immunoprecipitated proteins were separated on SDS-PAGE and western blot analysis was performed as described. Each Co-IP experiment was confirmed via reciprocal IP.

Statistical analysis. Four independent in vitro experiments were carried out. Statistical results were expressed as the mean \pm the standard deviation of the means obtained from triplicates of each independent experiment. Statistical significance of differences was determined by the paired Kruskal-Wallis non-parametric test.

\section{Results}

Cerulenin induces apoptosis in ZR-75-1 cells. Cerulenin at 25-55 $\mu \mathrm{M}$ efficiently reduced the viability of ZR-75-1 cells in a dose-dependent manner (Fig. 1A). Since the dose required for half-maximal inhibition of viability in ZR-75-1 cells was $45 \mu \mathrm{M}$ for cerulenin (Fig. 1B), this single concentration was utilized for further study. To prove that cerulenin reduced the viability of ZR-75-1 cells by inducing apoptosis, Hoechst staining and flow cytometry analysis were undertaken. A simultaneous estimation of cell cycle parameters and apoptosis by flow cytometry demonstrated that cerulenin increased the subdiploid apoptotic population in ZR-75-1 cells (Fig. 2A). Hoechst staining showed that cells treated cerulenin had fragmented atypical nuclei (Fig. 2B). Western blot assay of cerulenin-treated ZR-75-1 cells showed the degradation of caspase-3 (32 kDa) proform, the production of caspase- 3 cleavage products, and the downregulation of Bcl-2 protein expression (Fig. 2C). Flow cytometry revealed that the portion of cells showing the depolarization

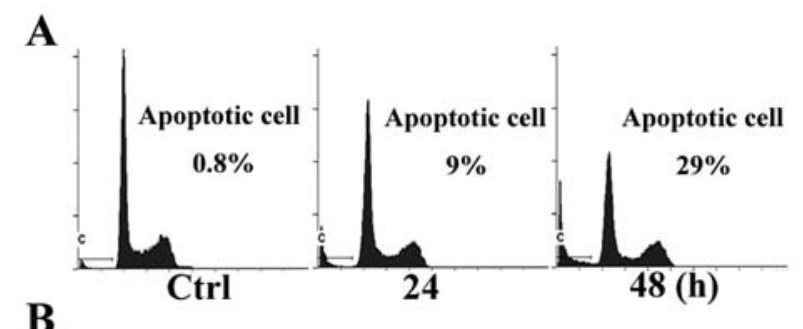

B

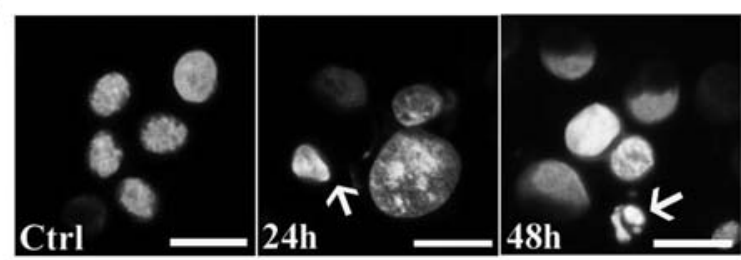

C

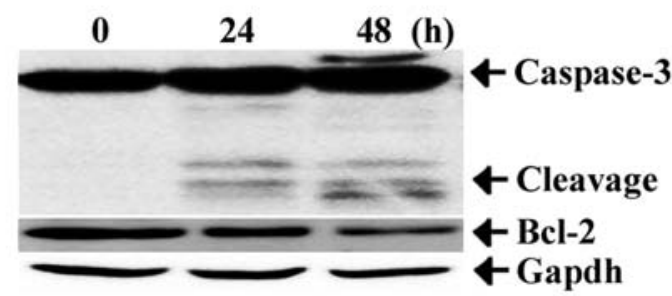

D

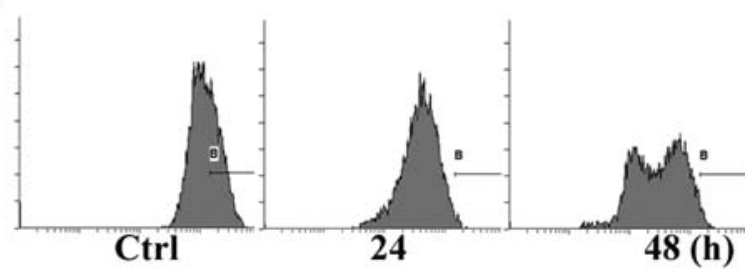

Figure 2. Cerulenin induces caspase-3-mediated apoptosis in ZR-75-1. (A Representative histograms show cell cycle progression and increase of DNA hypoploidy. (B) Cells treated with $45 \mu \mathrm{M}$ cerulenin underwent morphological changes characteristic of programmed cell death, including chromatin condensation and nuclear fragmentation. Bar, $20 \mu \mathrm{m}$. (C) Degradation of caspase-3 and production of their cleavage products, and downregulation of Bcl-2 was demonstrated in ZR-75-1 cells treated with cerulenin. (D) Flow cytometry data shows the reduction of mitochondrial membrane potential in cells undergoing apoptosis. Mitochondrial retention of DiOC6 decreased in cerulenin-treated cells.

of MMP was increased in ZR-75-1 cells after cerulenin treatment (Fig. 2D). Confocal microscopy also demonstrated that cerulenin treatment induced the release of cytochrome $\mathrm{c}$ from mitochondria in ZR-75-1 cells (Fig. 3A). Western blot assay of cerulenin-treated ZR-75-1 cells also showed that release of cytochrome $\mathrm{c}$ and AIF from mitochondria (Fig. 3B). These results indicate that cerulenin induces apoptosis in ZR-75-1 cells in a caspase- and mitochondria-dependent fashion.

Phospho-Akt and HKII accumulate in mitochondria upon cerulenin treatment. We next asked whether HKII is involved in the inhibition of FASN in human breast cancer ZR-75-1 cells. Since HKII possesses consensus sequences for phosphorylation by Akt (22) and Akt activation is modulated by FASN activity (11), subcellular localization of phosphorylated Akt as well as its protein level were assessed after cerulenin treatment. We examined whether phospho-Akt translocates to other cell compartments, in addition to its conventionally reported nuclear localization. 
A

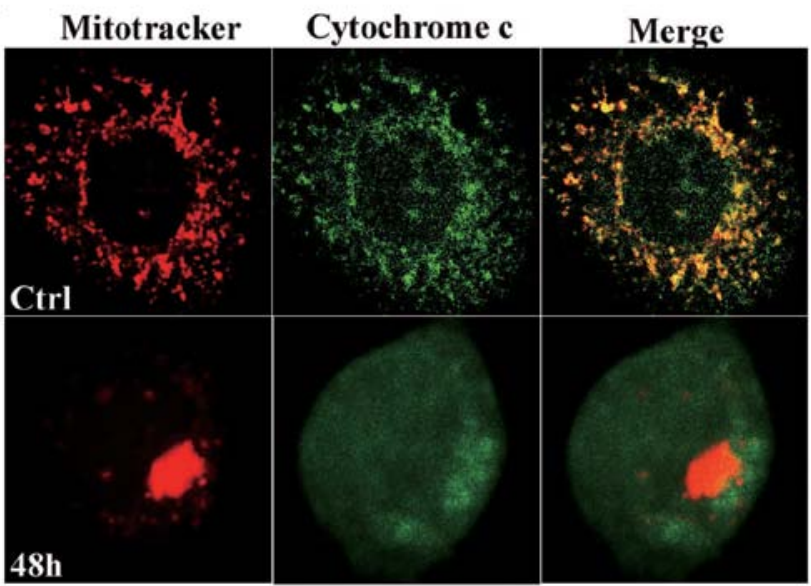

B

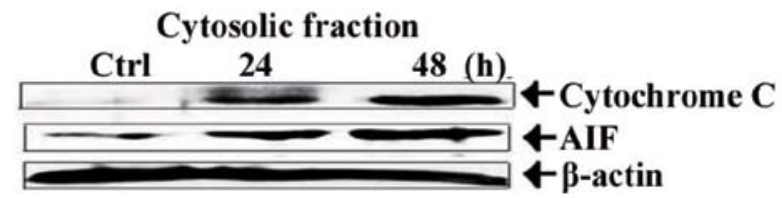

Figure 3. Cerulenin induces the mitochondrial release of cytochrome $\mathrm{c}$ and AIF. (A) Mitochondria were labeled by incubation of cells with $450 \mathrm{nM}$ Mitotracker Red for $30 \mathrm{~min}$ at $37^{\circ} \mathrm{C}$ before fixation and visualized by confocal microscopy. (B) Cells were treated with $45 \mu \mathrm{M}$ cerulenin, fractionated into mitochondrial and cytosolic fractions and blotted with cytochrome $\mathrm{c}$ and AIF antibodies.

We conducted cellular subfractionation studies in order to isolate enriched mitochondria fraction from untreated or cerulenin-treated ZR-75-1 cells. Then the mitochondria fraction and cytosolic fraction were loaded on gel and analyzed using western blot analysis (Fig. 4A). Putative contamination of the mitochondrial fractions was monitored by detecting mitochondrial (VDAC) and cytosolic ( $\beta$-actin) marker proteins. Notably, the phospho-Akt and HKII located in the mitochondrial fraction increased following cerulenin treatment. Confocal microscopical study also showed that phospho-Akt and HKII redistributes to mitochondria (Fig. 4B). The protein levels of FASN and phosphorylated Akt were downregulated while total Akt and HKII did not show significant changes with cerulenin treatment (Fig. 5A).

Protein-protein interaction between HKII and AIF was disrupted by cerulenin. The possibility that AIF may play a critical role in cerulenin-induced apoptosis by controlling its physical association with HKII, was tested by co-immunoprecipitation experiments. ZR-75-1 cells treated with cerulenin were lysed in RIPA lysis buffer and co-immunoprecipitation was carried out using anti-AIF antibody. The IP pulled-down samples were immunoblotted for both HKII and AIF. As shown in Fig. 5B, there was a detectable physical interaction between HKII and AIF in untreated control cells. Importantly, treatment of cells with $45 \mu \mathrm{M}$ cerulenin considerably disrupted such interaction.

Inhibition of PI3K/Akt by LY294002 sensitizes ZR-75-1 breast cancer cells to cerulenin-induced apoptosis. Increasing concentrations of LY294002 caused a gradual decrease in phospho-Akt and FASN protein levels (Fig. 6A). At concentrations that
A

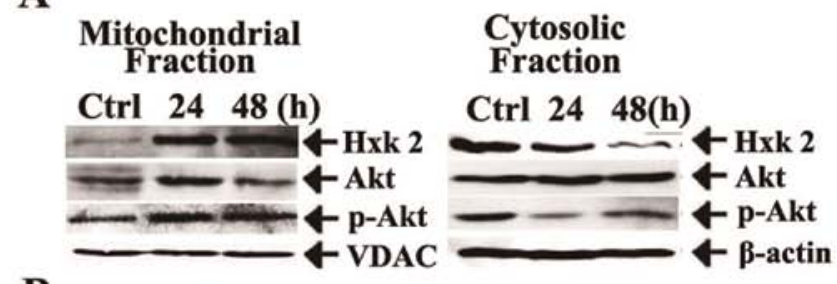

B
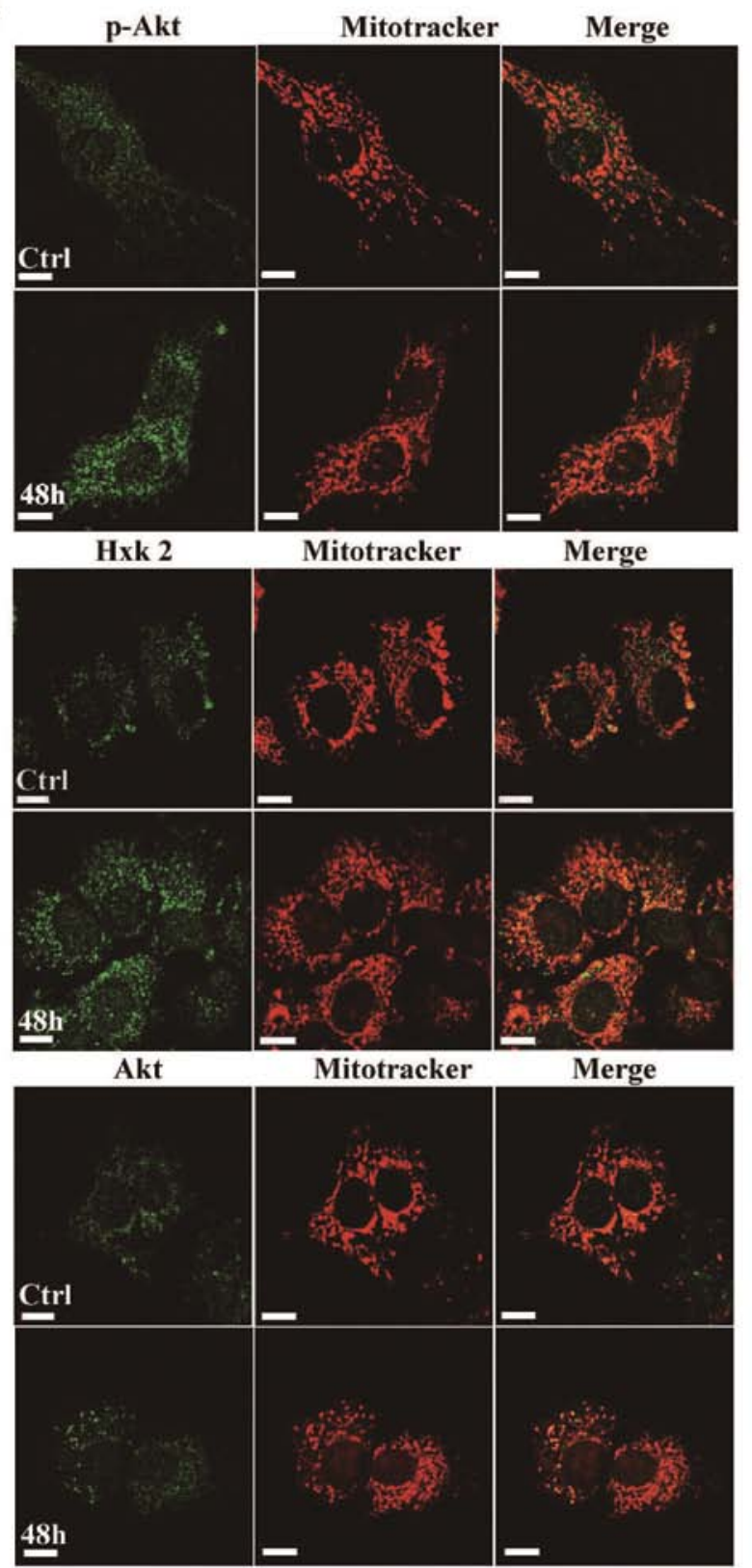

Figure 4. Phosphorylated Akt and HKII redistributes to mitochondria. (A) Cells were fractionated into mitochondrial and cytosolic fractions and blotted with VDAC, $\beta$-actin, HKII, phosphorylated Akt and total Akt antibodies. VDAC and $\beta$-actin were used as mitochondrial and cytosolic markers, respectively. (B) Cells were treated with $45 \mu \mathrm{M}$ cerulenin and the localization of Akt, phosphorylated Akt (p-Akt) and HKII (Hxk 2) were analysed using confocal microscopy.

significantly blocked activation of Akt $(40 \mu \mathrm{M})$, no overt signs of cellular toxicity or apoptosis were observed (Fig. 6B and E). Because LY294002 reduced the FASN level in ZR-75-1 cells, we reasoned that there could be a synergy in inducing apop- 
A

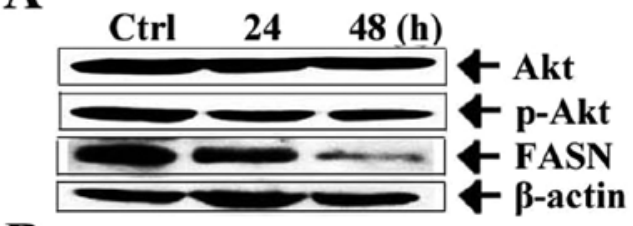

B

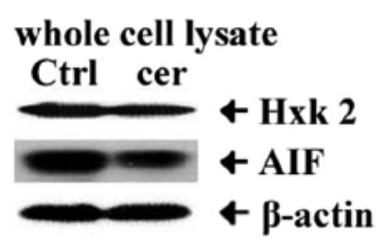

IP: AIF

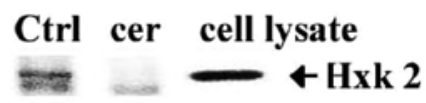

Figure 5. Physical interaction between HKII and AIF is disrupted by cerulenin treatment. (A) After $45-\mu \mathrm{M}$ cerulenin treatment, total cellular extracts were made. Equal amounts of protein were subjected to western blot analysis. (B) Cells were treated with $45 \mu \mathrm{M}$ cerulenin for $48 \mathrm{~h}$, and co-immunoprecipitation was carried out using AIF antibody. The IP products were immunoblotted for HKII. tosis when LY294002 was combinded with the FASN inhibitor cerulenin. As shown in Fig. 6C, treatment of ZR-75-1 with 45 $\mu \mathrm{M}$ cerulenin efficiently induced cell death in a time-dependent manner, whereas treatment with LY294002 (0-40 $\mu \mathrm{M})$ alone resulted in no significant cell death. Noticeably, co-treatment with $45 \mu \mathrm{M}$ cerulenin and $40 \mu \mathrm{M}$ LY294002 augmented cell death compared to cerulenin alone (Fig. 6D). The synergistic cytotoxic effect of cerulenin and LY294002 was confirmed in flow cytometry using propidium iodide (Fig. 6E). We further examined activation of caspase upon co-treatment of cerulenin and LY294002. Although LY294002 alone did not induce caspase-3 activation (Fig. 7A), co-treatment with cerulenin and LY294002 augmented the activation of caspase-3 in ZR-75-1 cells compared to cerulenin alone (Fig. 7B). Co-treatment with cerulenin and LY294002 also augmented the reduction of phospho-Akt level compared to cerulenin alone (Fig. 7C). However, cotreatment with cerulenin and LY294002 did not affect total Akt protein levels, indicating that Akt protein was not degraded upon this co-treatment (Fig. 7C). Co-treatment with cerulenin and LY294002 also augmented the reduction of FASN expression level compared to cerulenin alone. Co-treatment with cerulenin and LY294002 also augmented

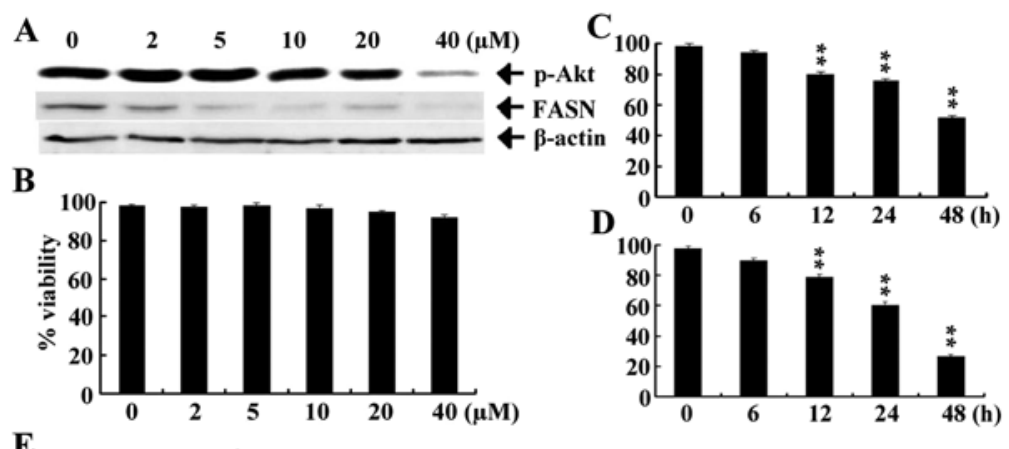

$\mathbf{E}$

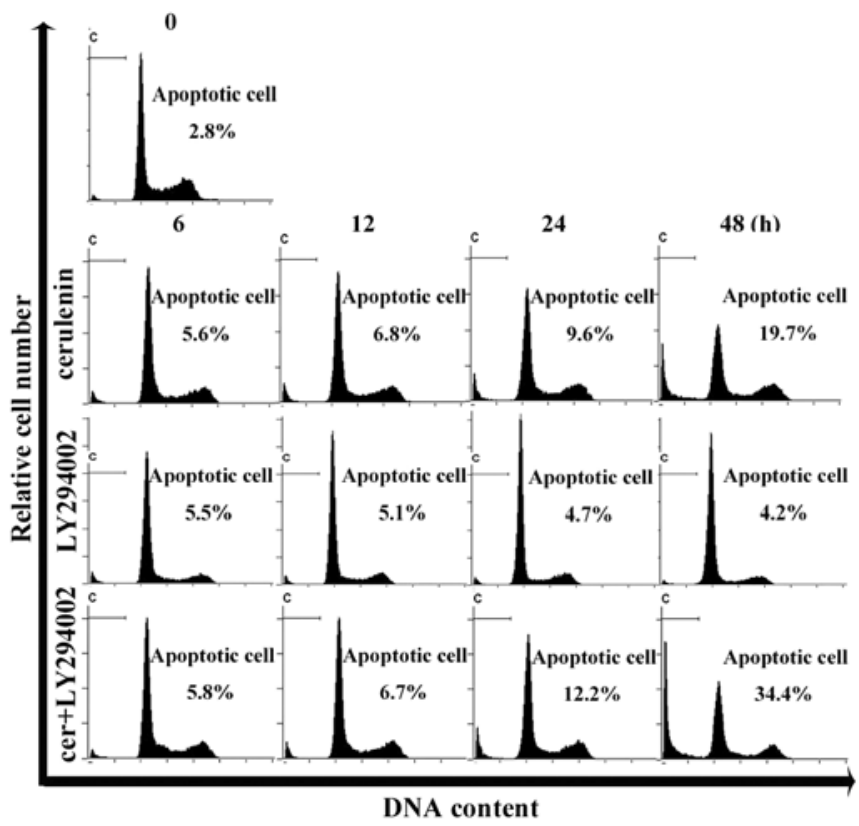

Figure 6. LY294002 sensitizes ZR-75-1 cells to cerulenin-induced apoptosis. (A and B) Cells were treated with LY294002 alone at the indicated concentrations for $48 \mathrm{~h}$. After $48 \mathrm{~h}$, total cellular extracts were prepared. Equal amounts of protein were subjected to western blot analysis. Viability was determined at the same time-point. (C and D) Cells were treated with $45 \mu \mathrm{M}$ cerulenin alone (C) or co-treated with $40 \mu \mathrm{M} \mathrm{LY924002} \mathrm{and} 45 \mu \mathrm{M}$ cerulenin (D) for 6, 12,24 and $48 \mathrm{~h}$ and the viability was determined by trypan blue exclusion assay. ${ }^{* *} \mathrm{P}<0.01$ versus untreated control. (E) Cells were treated with $45 \mu \mathrm{M}$ cerulenin alone, $40 \mu \mathrm{M}$ LY294002 alone or combination of cerulenin and LY294002 for the indicated times. Cells were harvested and fluorescence-activated cell sorting analysis was carried out using propidium iodide. 

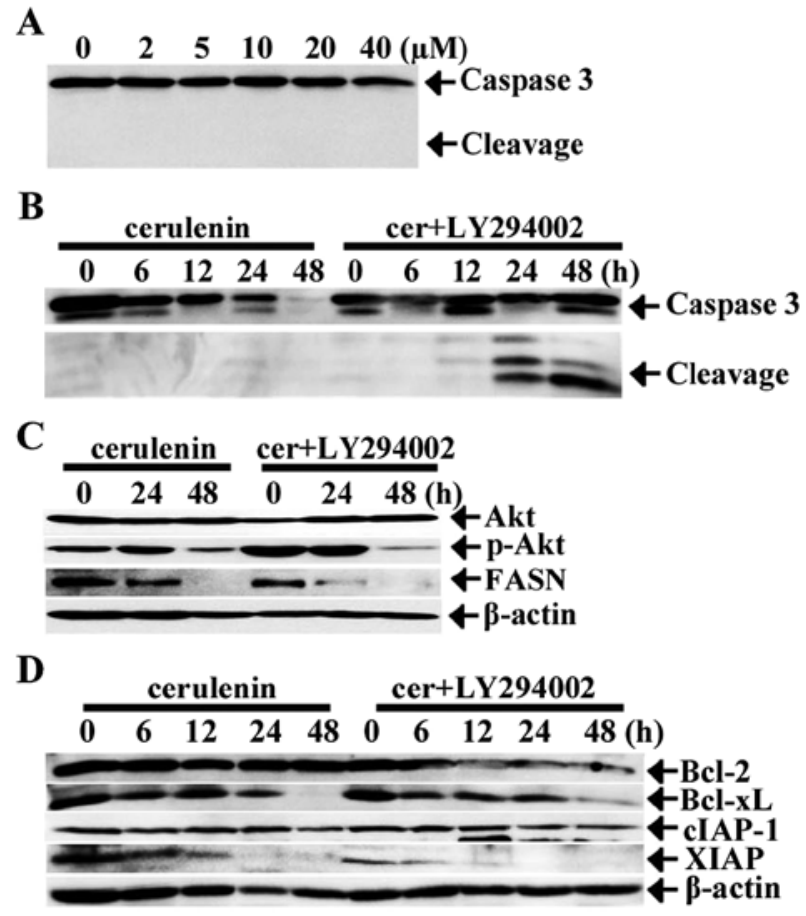

Figure 7. Co-treatment of LY294002 and cerulenin causes early caspase-3 activation and degradation of antiapoptotic proteins. (A) Cells were treated with LY294002 at the indicated concentrations for $48 \mathrm{~h}$. (B-D) Cells were treated with $45 \mu \mathrm{M}$ cerulenin alone or with $40 \mu \mathrm{M}$ LY294002 for the indicated times. Cells were then harvested and equal amounts of protein were used for western blot analysis.

the reduction of anti-apoptotic factors such as $\mathrm{Bcl}-2, \mathrm{Bcl}-\mathrm{xL}$, cIAP-1 and XIAP expression level compared to cerulenin alone (Fig. 7D). Collectively, these data show that LY294002 synergistically enhance apoptosis-inducing activity of cerulenin in ZR-75-1 breast cancer cells.

\section{Discussion}

Although FASN is highly expressed in most human cancers and holds promise as a cancer marker and as a target for antineoplastic therapy, we know very little of the molecular events responsible for overexpression of this key metabolic enzyme in cancer cells (13). In this study, we demonstrated that cerulenin at usual doses induces apoptosis in ZR-75-1 cells.

The tumor suppressor gene PTEN, encoding a dual-specificity phosphatase, has been cloned and mapped to chromosome $10 q 23.3$ (25). Loss of heterozygosity ( $\mathrm{LOH})$ of markers at 10q23-25 occurs frequently (30-50\%) in endometrial (26), glioblastoma (27), and breast cancer (28). Breast cancer cell lines have shown that a large proportion have biallelic loss of PTEN (25,29). While structural deletion or mutation of PTEN can lead to decreased PTEN protein levels, other mechanisms which lead to complete loss of PTEN expression seem to have a considerable proportion as well, at least in the breast carcinoma model (30). ZR-75-1, with a hemizygous deletion of PTEN and a missense mutation in the remaining allele, expresses low level of PTEN protein, yielding a weak band of the expected size (30). It has been well accepted that PTEN signals down-regulate the PI3K-Akt pathway and that PTEN inversely correlates with phospho-Akt (31).
Although it is now well established that Akt provides a potent survival signal in many cell types, the mechanisms for Akt-mediated survival have not been fully elucidated (9). In the unstimulated cell, Akt is mostly localized in the cytoplasmic compartment (22). Akt redistributes to mitochondria upon its activation and that it binds to and phosphorylates HKII, concomitantly preventing $\mathrm{Ca}^{2+}$ or $\mathrm{H}_{2} \mathrm{O}_{2}$-induced mitochondrial depolarization and cytochrome c release (22). HKII serves as a substrate of Akt phosphorylation (22). Mitochondrial-bound HKII activity is increased in cells treated with agonists known to activate Akt $(16,33)$. Recent studies have demonstrated the protective role of mitochondrial HKII in preservation of mitochondrial integrity $(9,16)$. Thus HKII seems to be a prospective target in Akt-mediated inhibition of mitochondrial damage (22).

The association of hexokinase with mitochondria has emerged as a powerful mechanism in protecting numerous cell types against cell death (33). However, our result shows that ZR-75-1 cells undergo apoptosis even though HKII accumulates in the mitochondria upon cerulenin treatment. Noticeably, we observed physical interaction between HKII and AIF in untreated control cells and disrupting this protein complex by cerulenin leads to release of AIF from the mitochondria and eventual cell death. Thus, we assume that while the observed mitochondrial redistribution does not contribute to cell survival, the physical interaction between HXII and AIF might be an important fail-safe mechanism to ensure cell death. AIF is known as a killer protein, which is important in mitochondriamediated cell death. AIF translocates from mitochondria to the nucleus and cause DNA condensation and fragmentation (32). However, much more study will be required to elucidate the biological function of AIF in the mitochondria and its regulation. It would be intriguing to investigate if HKII can regulate mitochondria function via changing its interaction with AIF.

Our data show that cerulenin results in the downregulation of phospho-Akt and redistributes phospho-Akt to mitochondria in ZR-75-1 cells. It is well known that mitochondria play a pivotal role in cerulenin-induced apoptosis in many cancer cell lines (34). Prior evidence has demonstrated that the nucleus is a target for activated Akt (35). A study using a FRET-based Akt activity reporter in NIH3T3 cells clearly shows that activated Akt detaches from the plasma membrane and translocates to other compartments including the nucleus (36). Thus, it appears that activated Akt translocates to several cellular compartments, including mitochondria and the nucleus, to execute acute and chronic protective effects on cells (22). A recent study also shows that activated Akt translocates to mitochondria and phosphorylates mitochondrial HKII and that Akt activation increased the total amount of HKII found in the mitochondrial fraction (36). Additionally, the study showed that association of HKII with mitochondria inhibits PT-pore induced cytochrome c release, and overexpression of HKII confers protection against stress (36). Further future study is required to elucidate the mechanisms by which HKII modulates PT pore opening.

Cancer indicates a disease that is distinguished by multiple genetic defects, epigenetic changes, and plasticity in response to its environment (4). Therefore, multitarget therapy is inevitable to acquire optimal efficacy (4). Treatment with $40 \mu \mathrm{M}$ LY294002 alone for $6 \mathrm{~h}$ is sufficient to induce apoptosis in LNCaP prostate cancer cells $(13,37)$, however, it is insufficient 
to induce apoptosis in ZR-75-1 breast cancer cells even after $48 \mathrm{~h}$ (Fig. 6B and E). These data indicate that both of Akt and FASN contribute to ZR-75-1 cell survival. Therefore, this study demonstrating the sensitization of cerulenin-induced apoptosis by LY294002 provides a scientific rationale for a combination therapy of a FASN inhibitor and PI3K/Akt pathway inhibitor (4). Co-treatment of ZR-75-1 cells with cerulenin and LY294002 induces cytochrome $c$ release and the subsequent caspase activation; the activated caspase causes the degradation of the antiapoptotic proteins such as XIAP which induces more cell death compared with cell death induced by cerulenin itself (4).

In conclusion, cerulenin induces apoptosis in ZR-75-1 cells by downregulating phosphorylated Akt and disrupting interaction between HKII and AIF. Furthermore, co-treatment with cerulenin and LY294002 sensitizes ZR-75-1 cells to apoptosis. It remains an open question through which exact molecular mechanism regulates the cerulenin-induced apoptosis in ZR-75-1 cells, and it may be anticipated that the answer to the question will yield further insights into the mechanism of the resistance conferred by Akt and HKII and the development of therapeutic strategy to overcome the resistance.

\section{Acknowledgements}

This study was supported by Basic Science Research Program through the National Research Foundation of Korea (NRF) funded by the Ministry of Education, Science, and Technology (2010-0001942), Republic of Korea.

\section{References}

1. Kuhajda FP: Fatty-acid synthase and human cancer: new perspectives on its role in tumor biology. Nutrition 16: 202-208, 2000.

2. Baron A, Migita T, Tang D and Loda M: Fatty acid synthase: a metabolic oncogene in prostate cancer? J Cell Biochem 91: 47-53, 2004.

3. GanslerTS, Hardman III W, Hunt DA, Schaffel S and Hennigar RA: Increased expression of fatty acid synthase (OA-519) in ovarian neoplasms predicts shorter survival. Hum Pathol 28: 686-692, 1997.

4. Liu X, Shi Y, Giranda VL and Luo Y: Inhibition of the phosphatidylinositol 3-kinase/Akt pathway sensitizes MDA-MB468 human breast cancer cells to cerulenin-induced apoptosis. Mol Cancer Ther 5: 494-501, 2006.

5. PizerES,ChrestFJ,DiGiuseppe JA and Han WF: Pharmacological inhibitors of mammalian fatty acid synthase suppress DNA replication and induce apoptosis in tumor cell lines. Cancer Res 58: 4611-4615, 1998

6. De Schrijver E, Brusselmans K, Heyns W, Verhoeven G and Swinnen JV: RNA interference-mediated silencing of the fatty acid synthase gene attenuates growth and induces morphological changes and apoptosis of $\mathrm{LNCaP}$ prostate cancer cells. Cancer Res 63: 3799-3804, 2003.

7. Gabrielson EW, Pinn ML, Testa JR and Kuhajda FP: Increased fatty acid synthase is a therapeutic target in mesothelioma. Clin Cancer Res 7: 153-177, 2001.

8. Kuhajda FP, Jenner K, Wood FD, Hennigar RA, Jacobs LB and Dick JD: Fatty acid synthesis: a potential selective target for antineoplastic therapy. Proc Natl Acad Sci USA 91: 6379-6383, 1994.

9. Pizer ES, Wood FD, Heine HS, Romantsev FR, Pasternack GR and Kuhajda FP: Inhibition of fatty acid synthesis delays disease progression in a xenograft model of ovarian cancer. Cancer Res 56: 1189-1193, 1996.

10. Vivanco I and Sawyers CL: The phosphatidylinositol 3-kinase AKT pathway in human cancer. Nat Rev Cancer 2: 489-501, 2002.
11. Wang HQ, Altomare DA, Skele KL, Poulikakos PI, Kuhajda FP, Di Cristofano A and Testa JR: Positive feedback regulation between AKT activation and fatty acid synthase expression in ovarian carcinoma cells. Oncogene 24: 3574-3582, 2005.

12. Yang YA, Han WF, Morin PJ, Chrest FJ and Pizer ES: Activation of fatty acid synthesis during neoplastic transformation: role of mitogen-activated protein kinase and phosphatidylinositol 3-kinase. Exp. Cell Res 279: 80-90, 2002.

13. Van de Sande T, De Schrijver E, Heyns W, Verhoeven G and Swinnen JV: Role of the phosphatidylinositol 3'-kinase/PTEN/ Akt kinase pathway in the overexpression of fatty acid synthase in LNCaP prostate cancer cells. Cancer Res 62: 642-646, 2002.

14. Wang D and Sul HS: Insulin stimulation of the fatty acid synthase promoter is mediated by the phosphatidylinositol 3-kinase pathway. Involvement of protein kinase B/Akt. J Biol Chem 273: 25420-25426, 1998.

15. del Peso L, González-García M, Page C, Herrera R and Nuñez G: Interleukin-3-induced phosphorylation of BAD through the protein kinase Akt. Science 278: 687-689, 1997.

16. Majewski N, Nogueira V, Bhaskar P, Coy PE, Skeen JE, Gottlob K, et al: Hexokinase mitochondria interaction mediated by Akt is required to inhibit apoptosis in the presence or absence of Bax and Bak. Mol Cell 16: 819-830, 2004.

17. Robey RB and Hay N: Mitochondrial hexokinases, novel mediators of the antiapoptotic effects of growth factors and Akt. Oncogene 25: 4683-4696, 2006

18. Mathupala SP, Ko YH and Pedersen PL: Hexokinase II: cancer's double-edged sword acting as both facilitator and gatekeeper of malignancy when bound to mitochondria. Oncogene 25: 4777-4786, 2006.

19. Wilson JE: Isozymes of mammalian hexokinase: structure, subcellular localization and metabolic function. J Exp Biol 206: 2049-2057, 2003.

20. Cardenas ML, Cornish-Bowden A and Ureta T: Evolution and regulatory role of the hexokinases. Biochim Biophys Acta 1401: 242-264, 1998.

21. Pastorino JG, Hoek JB and Shulga N: Activation of glycogen synthase kinase $3 \mathrm{~b}$ disrupts the binding of hexokinase II to mitochondria by phosphorylating voltage-dependent anion channel and potentiates chemotherapy-induced cytotoxicity. Cancer Res 65: 10545-10554, 2005.

22. Miyamoto S, Murphy AN and Brown JH: Akt mediates mitochondrial protection in cardiomyocytes through phosphorylation of mitochondrial hexokinase-II. Cell Death Differ 15: 521-529, 2008.

23. Gottlob K, Majewski N, Kennedy S, Kandel E, Robey RB and Hay N: Inhibition of early apoptotic events by Akt/PKB is dependent on the first committed step of glycolysis and mitochondrial hexokinase. Genes Dev 15: 1406-1418, 2001.

24. Pizer ES, Wood FD, Pasternack GR and Kuhajda FP: Fatty acid synthase (FAS): a target for cytotoxic antimetabolites in HL6O promyelocytic leukemia cells. Cancer Res 4: 745-751, 1996.

25. Steck PA, Pershouse MA, Jasser SA, Yung WK, Lin H, Ligon AH, Langford LA, Baumgard ML, Hattier T, Davis T, Frye C, Hu R, Swedlund B, Teng DH and Tavtigian SV: Identification of a candidate tumor suppressor gene, MMAC1, at chromosome $10 \mathrm{q} 23.3$ that is mutated in multiple advanced cancers. Nat Genet 15: 356-362, 1997.

26. Kong D, Suzuki A, Zou TT, Sakurada A, Kemp LW, Wakatsuki S, Yokoyama T, Yamakawa H, Furukawa T, Sato M, Ohuchi N, Sato S, Yin J, Wang S, Abraham JM, Souza RF, Smolinski KN, Meltzer SJ and Horii A: PTEN1 is frequently mutated in primary endometrial carcinomas. Nat Genet 17: 143-144, 1997.

27. Maier D, Zhang ZW, Taylor E, Hamou MF, Gratzl O, van Meir EG, Scott RJ and Merlo A: Somatic deletion mapping on chromosome 10 and sequence analysis of PTEN/MMAC1 point to the 10q25-26 region as the primary target in low-grade and high-grade gliomas. Oncogene 16: 3331-3335, 1998.

28. Bose S, Wang SI, Terry MB, Hibshoosh H and Parsons R: Allelic loss of chromosome 10q23 is associated with tumor progression in breast carcinomas. Oncogene 17: 123-127, 1998.

29. Li J, Yen C, Liaw D, Podsypanina K, Bose S, Wang S, Puc J, Miliaresis C, Rodgers L, McCombie R, Bigner SH, Giovanella BC, Ittman M, Tycko B, Hibshoosh H, Wigler MH and Parsons R: PTEN, a putative protein tyrosine phosphatase gene mutated in human brain, breast, and prostate cancer. Science 275: 1943-1947, 1997. 
30. Perren A, Weng LP, Boag AH, Ziebold U, Thakore K, Dahia PL, Komminoth P, Lees JA, Mulligan LM, Mutter GL and Eng C: Immunohistochemical evidence of loss of PTEN expression in primary ductal adenocarcinomas of the breast. Am J Pathol 155 : 1253-1260, 1999.

31. Stambolic V, Suzuki A, de la Pompa JL, Brothers GM, Mirtsos C, Sasaki T, Ruland J, Penninger JM, Siderovski DP and Mak TW: Negative regulation of $\mathrm{PKB} / \mathrm{Akt}-$ dependent cell survival by the tumor suppressor PTEN. Cell 95: 29-39, 1998.

32. Urbano A, Lakshmanan U, Choo PH, Kwan JC, Ng PY, Guo K, Dhakshinamoorthy S and Porter A: AIF suppresses chemical stress-induced apoptosis and maintains the transformed state of tumor cells. EMBO J 24: 2815-2826, 2005.

33. Majewski N, Nogueira V, Robey RB and Hay N: Akt inhibits apoptosis downstream of BID cleavage via a glucose-dependent mechanism involving mitochondrial hexokinases. Mol Cell Biol 24: $730-740,2004$.
34. Heiligtag SJ, Bredehorst R and David KA: Key role of mitochondria in cerulenin-mediated apoptosis. Cell Death Differ 9: 1017-1025, 2002.

35. Kunkel MT, Ni Q, Tsien RY, Zhang J and Newton AC: Spatiotemporal dynamics of protein kinase B/Akt signaling revealed by a genetically encoded fluorescent reporter. J Biol Chem 280: 5581-5587, 2005.

36. Bryson JM, Coy PE, Gottlob K, Hay N and Robey RB: Increased hexokinase activity, of either ectopic or endogenous origin, protects renal epithelial cells against acute oxidant-induced cell death. J Biol Chem 277: 11392-11400, 2002.

37. Lin J, Adam R, Santiestevan E, and Freeman M: The phosphatidylinositol 3'-kinase pathway is a dominant growth factor-activated cell survival pathway in LNCaP human prostate carcinoma cells. Cancer Res 59: 2891-2897, 1999. 